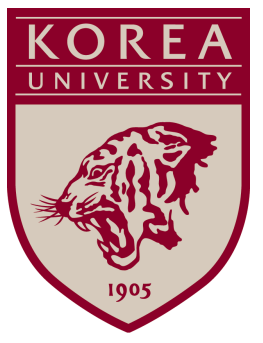

Discussion Paper Series

No. 1304

Nov 2013

\title{
Auctions with imperfect commitment when the reserve may serve as a signal
}

\author{
Byoung Heon Jun and Elmar G. Wolfstetter
}

The Institute of Economic Research - Korea University

Anam-dong, Sungbuk-ku, Seoul, 136-701, South Korea, Tel: (82-2) 3290-1632, Fax: (82-2) 928-4948

Copyright (C) 2013 IER. 


\title{
Auctions with imperfect commitment when the reserve may serve as a signal*
}

\author{
Byoung Heon Jun ${ }^{\dagger}$ \\ Korea University, Seoul \\ Department of Economics
}

\author{
Elmar G. Wolfstetter ${ }^{\ddagger}$ \\ Humboldt-University at Berlin and \\ Korea University, Seoul
}

November 4, 2013

\begin{abstract}
If bidders are uncertain whether the auctioneer sticks to the announced reserve, some bidders respond by strategic non-participation, speculating that the auctioneer may revoke the reserve. However, the reserve inadvertently signals the auctioneer's type, which drives a unique separating and a multitude of pooling equilibria. If one eliminates belief systems that violate the "intuitive criterion", one obtains a unique equilibrium reserve price equal to the seller's own valuation. Paradoxically, even if bidders initially believe that the auctioneer is bound by his reserve almost with certainty, commitment has no value.
\end{abstract}

KEYWORDs: Auctions, mechanism design.

JEL Classifications: D21, D43, D44, D45.

\section{Introduction}

Anecdotal evidence indicates that some auctioneers do not always stick to the announced rules and occasionally revoke the reserve if no bids are forthcoming. Typically, this action ignites bidding and it is not uncommon that the closing bid exceeds the initial reserve. This suggests that bidders anticipate the auctioneer's lack of perfect commitment and sometimes take a gamble and forgo meeting the reserve, hoping that the reserve is revoked and a bargain may be struck at a price below the reserve, while risking no trade.

Whereas this behavior is endemic in low to medium caliber auctions ${ }^{1}$, major auction houses tend to stick to the stipulated reserve. However, they have a similar tendency to bypass their rules by discreetly re-auctioning objects that did not meet the reserve. Such behavior is reported repeatedly by those who keep track of the performance of major auction houses like Christie's and Sotheby's. ${ }^{2}$

In the present paper, we examine this behavior in auctions, focusing on open ascending bid (English) auctions. For this purpose we first analyze the equilibrium behavior of bidders who believe that

${ }^{*}$ Research support by the National Research Foundation of Korea funded by the Korean Government (NRF-2010330-B00085), and the Deutsche Forschungsgemeinschaft (DFG), SFB Transregio 15, "Governance and Efficiency of Economic Systems" is gratefully acknowledged.

${ }^{\dagger}$ Email: bhjun@korea.ac.kr

${ }^{\ddagger}$ Corresponding Author: Institute of Economic Theory I, Humboldt University at Berlin, Spandauer Str. 1, 10178 Berlin, Germany, Email: wolfstetter@gmail.com

${ }^{1} \mathrm{~A}$ case in point is the annual Christmas Art Auction of the Berlin Senate. There, the city government auctions the work of artists who were supported by a fellowship. In these auctions, the auctioneer occasionally initiated the breach of his rules with the words "Just once, I revoke the reserve.".

${ }^{2}$ For example, McAfee and Vincent (1997, p. 246) report: "Regular participants in the now defunct Christies' auctions of fine wines often experience déjà vu. The same bottles or rare wine seemed to appear auction after auction." In order to understand this behavior one must keep in mind that auction houses are agents of the seller and they have to act in the interest of the seller, even though this may impair their reputation. 
the auctioneer revokes the reserve if it is not met, with some probability. Not surprisingly, we find that bidders forgo bidding and speculate on a revocation of the reserve if and only if their valuation is relatively close to the initial reserve. For high valuation bidders it does not pay to take the risk of foregoing trade by not meeting the reserve.

However, bidders' beliefs cannot be arbitrary; they must be supported by rationally predicted behavior of the auctioneer. This brings us to the second ingredient of our analysis: the fact that the reserve price may inadvertently signal the auctioneer's type. Bidders have prior non-degenerate beliefs concerning the type of auctioneer, and update their beliefs after observing the reserve set by the auctioneer. If, in equilibrium, the two types of auctioneer set different reserves, bidders can perfectly infer the auctioneer's type after observing the reserve; whereas if they "pool" and set the same reserve, beliefs remain unchanged.

Like in standard signaling games where signals are proactively used to reveal one's type, the present game admits a multitude of equilibria that exhibit either pooling (same reserves) or full revelation (distinct reserves). This multitude is of course due to the arbitrariness of "off-equilibrium" beliefs, where Bayes' rule cannot be applied to assure consistency of beliefs with equilibrium strategies. Therefore, one needs to eliminate implausible beliefs by applying well-known equilibrium refinements such as the "intuitive criterion" introduced by Cho and Kreps (1987) ${ }^{3}$

After applying the intuitive criterion the number of equilibria is drastically reduced. Only one pooling and one separating equilibrium survive. Interestingly, in the unique separating equilibrium, the auctioneer who is committed to never revoke his reserve price must set a reserve price equal to zero, and in the unique pooling equilibrium both types of auctioneers set a reserve equal to zero. Therefore, in both equilibria, commitment has no value. We thus arrive at the paradoxical conclusion that no matter how unlikely it is that the auctioneer lacks commitment, commitment has no value.

This finding is somewhat similar to a well-known paradox by Bagwell (1995) who observed in the framework of a duopoly game that imperfect observability of the first mover's actions tends to destroy the value of commitment. However, in the present paper the erosion of the value of commitment is not due to imperfect observability, and it is not restricted to pure strategy equilibria, because we also cover mixed equilibria. 4

Imperfect commitment in English auctions has also been studied by Caillaud and Mezzetti (2004) in the context of a sequence of two English clock auctions of two identical objects. They assume that the auctioneer cannot commit to a sequence of reserve prices, although he can commit to a reserve price in each of the two auctions. Subsequently, the auctioneer takes advantage of the information revealed by the outcome of the first auction to set the optimal reserve price in the second auction. In turn, bidders respond by not bidding in the first auction unless their valuation is sufficiently larger than the reserve price. Therefore, the equilibrium exhibits some pooling property.

A complementary issue has been pursued by McAfee and Vincent (1997) who consider an auctioneer who is not committed to stick to the announced reserve and who re-auctions objects after some lapse of time, if the reserve has not been met, and in each auction resets the optimal reserve, based on updated information about bidders' valuations. Similar to a well-known property of the durable goods monopoly they find that the reserve converges to the seller's own valuation, if the time span between auctions becomes arbitrarily small.

Another complementary issue is pursued in the auctions with resale literature, which assumes that the auctioneer cannot prevent bidders from reselling to each other, after the auction. This issue

\footnotetext{
13.4).

${ }^{3}$ For a detailed survey of various equilibrium refinements see Van Damme (1987) and Kreps $(1990$, Sects. 12.7,

${ }^{4}$ In Bagwell (1995), imperfect observability destroys the value of commitment in the pure strategy equilibrium of his game. However, his game has also two mixed strategy equilibria, in one of which the value of commitment is restored (see van Damme and Hurkens, 1997).
} 
comes up if the outcome of the primary auction is inefficient, which occurs notoriously if the primary auction is a first-price auction and bidders are asymmetric (see ???).$^{5}$

The plan of the paper is as follows: In Section 2 we spell out the assumptions. In Section 3 we analyze equilibrium bidding for given beliefs concerning the auctioneer's type, and in Section 4 we analyze the signaling equilibrium reserve prices, followed by a proof that a standard equilibrium refinement yields one unique separating and one unique pooling equilibrium (Section 5). The paper closes with a discussion in Section 6 .

\section{Model}

Consider an open, ascending-bid English clock auction. There, the auctioneer quotes a reserve price. A bid is an expressed willingness to buy the object at the stipulated price. If two or more bids are submitted at the given price, the auctioneer continuously increases that price until there is no excess demand. Once a bidder has failed to bid at a certain price, he is no longer permitted to bid at higher prices (activity rule).

In order to keep the analysis as simple as possible, we consider the case of two bidders whose valuations are i.i.d. random variables drawn from the continuous c.d.f. $F$ and positive p.d.f., $f$, with support $(0,1)$.

The auctioneer is either committed to stick to the announced reserve price $r$ (referred to type $t=c$ ) or he is not committed (type $n$ ) and free to restart the auction with a zero reserve price in the event when no bids are forthcoming.

Bidders have non-degenerate prior beliefs and assume that the auctioneer is not committed with probability $q_{0} \in(0,1)$. Bidders update their beliefs after observing the reserve price, applying Bayes' rule, if possible.

\section{Equilibrium bidding for given beliefs}

We first solve the bidding subgames that are played after the auctioneer has announced the reserve and bidders have updated their beliefs. Bidders beliefs are denoted by $q(r):=\operatorname{Pr}\{\tilde{t}=n \mid r\}$.

Of course, truthful bidding is a dominant strategy for those who bid. However, bidders "speculate" and strategically abstain from bidding even if their valuation is somewhat greater than the reserve price.

Proposition 1. Bidders play a symmetric equilibrium cutoff strategy: if $r \leq \hat{r}:=1-q+q E(X)$, bidders bid if and only if their valuation is greater or equal to the cutoff value $\gamma \leq 1$, which is implicitly defined as the solution of:

$$
r=(1-q) \gamma+q E(X \mid X \leq \gamma))
$$

and no one bids if $r>\hat{r}$ (unless the auction is restarted).

Proof. First, suppose bidders play a symmetric cutoff strategy with cutoff value $\gamma \leq 1$ (which we prove in Appendix A). Then, a bidder whose valuation is equal to the cutoff value $\gamma$ must be indifferent between bidding and not bidding. If that bidder wins, he pays $r$; if he does not bid and no one else bid and the auctioneer is type $n$, all bidders bid truthfully in the subsequent auction Therefore,

$$
\int_{0}^{\gamma}(\gamma-r) d F(y)=q \int_{0}^{\gamma}(\gamma-y) d F(y) .
$$

Rearranging yields condition (1).

\footnotetext{
${ }^{5} \mathrm{An}$ exception to this rule is Haile (2000), where resale is driven by updated information about valuations that arrives after the primary auction.
} 
Existence and uniqueness of $\gamma$ follow from the fact that the function $\psi(\gamma):=(1-q) \gamma+q E(X \mid X \leq$ $\gamma)$ ) is strictly increasing and equal to zero at $\gamma=0$ and equal to $\hat{r}$ at $\gamma=1$, so that for all $r \leq \hat{r}$ there must be a $\gamma \leq 1$ that solves equation (1).

Second, if $r>\hat{r}$, the condition of indifference (1) cannot be satisfied for $\gamma \leq 1$ and not bidding is an equilibrium because even a bidder with valuation equal to 1 is better off by not bidding if his rival does not bid, which follows from

$$
r>\hat{r} \Longleftrightarrow q \int_{0}^{1}(1-y) d F(y)>\int_{0}^{1}(1-r) d F(y) .
$$

Therefore, no one bids if $r>\hat{r}$ and $\gamma \leq 1$ otherwise.

The seller's expected revenues are: $:^{6}$

$$
\begin{aligned}
\pi_{c}(r, q) & =\int_{\gamma(r, q)}^{1} \int_{\gamma(r, q)}^{v} z f_{(12)}(v, z) d z d v+\int_{\gamma(r, q)}^{1} \int_{0}^{\gamma(r, q)} r f_{(12)}(v, z) d z d v \\
& =\int_{\gamma(r, q)}^{1} \int_{\gamma(r, q)}^{v} z f_{(12)}(v, z) d z d v+2 r F(\gamma(r, q))(1-F(\gamma(r, q))) \\
\pi_{n}(r, q) & =\pi_{c}(r, q)+\int_{0}^{\gamma(r, q)} \int_{0}^{v} z f_{(12)}(v, z) d z d v \\
& =\pi_{c}(r, q)+2 \int_{0}^{\gamma(r, q)} z(F(\gamma(r, q))-F(z)) f(z) d z .
\end{aligned}
$$

Lemma 1. Denote the second largest order static of the sample of the two i.i.d. valuations by $X_{(2: 2)}$. One has:

$$
\begin{gathered}
r>\hat{r} \Rightarrow \pi_{c}(r, q)=0 \quad \text { and } \quad \pi_{n}(r, q)=E\left(X_{(2: 2)}\right) \\
r=0 \Rightarrow \pi_{c}(r, q)=\pi_{n}(r, q)=E\left(X_{(2: 2)}\right) \\
q=1 \Rightarrow \pi_{n}(r, q)=E\left(X_{(2: 2)}\right)
\end{gathered}
$$

Proof. Assertion (5) follows from Proposition 1.

Obviously, $r=0 \Rightarrow \gamma=0$; using the fact that $2(1-F(y)) f(y)$ is equal to the p.d.f. of the second-highest order statistic, $X_{(2: 2)}$, this confirms (6).

The proof of (7) is in two steps: 1) by (1), $q=1 \Rightarrow r=E(X \mid X \leq \gamma)$. 2) Moreover, using 1):

$$
\begin{aligned}
2 r F(\gamma(r, 1))(1-F(\gamma(r, 1))) & =2 \int_{0}^{\gamma(r, 1)} z d F(z)(1-F(\gamma(r, 1)) \\
& =2 \int_{0}^{\gamma(r, 1)} z d F(z) \int_{\gamma(r, 1)}^{1} d F(v) \\
& =\int_{\gamma(r, 1)}^{1} \int_{0}^{\gamma(r, 1)} z f_{(12)}(z, v) d z d v
\end{aligned}
$$

Therefore, $\pi_{c}(r, 1)=\int_{\gamma(r, 1)}^{1} \int_{0}^{v} z f_{(12)}(z, v) d z d v, \pi_{n}(r, 1)=\int_{0}^{1} \int_{0}^{v} z f_{(12)}(z, v) d z d v=E\left(X_{(2: 2)}\right)$.

Result (7) indicates that type $n$ cannot benefit from using a positive reserve price if bidders believe that he is type $n$ with probability 1 because his payoff is the same as if he used a reserve price equal to zero. This property is an essential element of our analysis.

\footnotetext{
${ }^{6}$ The joint p.d.f. of the two order statistics is $f_{(12)}(v, z)=2 f(v) f(z)$ for $v \geq z$ and zero otherwise.
} 


\section{Equilibrium reserve price}

The game may have equilibria in pure and in mixed strategies. The equilibria in pure strategies are either separating or pooling. The equilibria in mixed strategies are either separating, which occurs if the supports of strategies are disjoint, or (partial) pooling, which occurs if the intersection of the supports is not empty. In the following we show which of these applies.

Proposition 2. 1) The game has no separating equilibrium (in pure or in mixed strategies) in which type c employs a positive reserve price, $r_{c}>0$. 2) However, there are separating equilibria with $r_{c}=0$ (and $r_{n}>0$ ); in these equilibria commitment has no value.

Proof. 1) Suppose the game has a separating equilibrium, $\left(r_{c}, r_{n}, \gamma(r, q), q(r)\right)$ with $r_{c}>0$. Then, one must have $q\left(r_{c}\right)=0, q\left(r_{n}\right)=1$, and

$$
E\left(X_{(2: 2)}\right)=\pi_{n}\left(r_{n}, q\left(r_{n}\right)\right) \geq \pi_{n}\left(r_{c}, q\left(r_{c}\right)\right)>\pi_{c}\left(r_{c}, q\left(r_{c}\right)\right) \geq \pi_{c}(0, q(0))=E\left(X_{(2: 2)}\right),
$$

which is a contradiction.

This argument applies also to separating mixed equilibria, where the supports of the seller's strategies are disjoint.

2) Consider $r_{c}=0, r_{n}>0$ and the belief system

$$
q(r)= \begin{cases}0 & \text { if } r=0 \\ 1 & \text { otherwise }\end{cases}
$$

Then, $\pi_{c}(r, 1) \leq \pi_{n}(r, 1)$, by (4). Therefore, given the belief system $q(r)$ type $c$ cannot benefit if he deviates from $r_{c}=0$. Moreover, at the given belief system type $n$ earns a payoff equal to $E\left(X_{(2: 2)}\right)$, for all $r$. Therefore, type $n$ cannot benefit if he deviates from $r=r_{n}$ either.

These results have an intuitive interpretation. Suppose the game had a separating equilibrium with $r_{n} \neq r_{c}>0$. Then, the equilibrium payoff of type $n$ is the same as if he used a zero reserve price, by Equation (7) in Lemma 1 whereas the equilibrium payoff of type $c$ is greater or equal to that of type $n$. In that case type $n$ benefits from mimicking $c$, because unlike type $c$ he can restart the auction if no bids are forthcoming. Therefore, this cannot be an equilibrium. The incentive to mimic type $c$ vanishes only if type $c$ sets a reserve price equal to zero, in which case any reserve price $r_{n}$ is a best reply of type $n$.

Essentially, the non-existence of a separating equilibrium with $r_{c}>0$ is due to the fact that type $n$ can mimic type $c$ and benefit from this at no cost.

Proposition 3. The game has pooling equilibria in pure strategies.

Proof. Assume the belief system $q(r)=1$ if $r=r^{*}$ and $q(r)=0$ otherwise. Then, one obtains a pooling equilibrium in which both types set the common reserve price $r^{*}$, for all $r^{*}$ for which

$$
\pi_{c}\left(r^{*}, q_{0}\right) \geq \pi_{c}\left(0, q_{0}\right)=E\left(X_{(2: 2)}\right) .
$$

In order to prove this, use the fact that for all $r \neq r^{*}, q(r)=1$ and:

$$
\pi_{n}\left(r^{*}, q_{0}\right) \geq \pi_{c}\left(r^{*}, q_{0}\right) \geq \pi_{c}\left(0, q_{0}\right)=E\left(X_{(2: 2)}\right)=\pi_{n}(r, 1) \geq \pi_{c}(r, 1) .
$$

Therefore, neither type $c$ nor type $n$ can benefit by deviating from $r=r^{*}$.

A particular pooling equilibrium is obtained for $r^{*}=0$, because obviously $\pi_{n}\left(0, q_{0}\right)=\pi_{c}\left(0, q_{0}\right)$.

Proposition 4. The game has no mixed strategy equilibrium in which the supports of strategies have more than one element in common. 
Proof. Suppose the intersection contains at least two elements, denoted by $r_{1}, r_{2}$. Then, both types must be indifferent between them, i.e.,

$$
\pi_{c}\left(r_{1}, q\left(r_{1}\right)\right)=\pi_{c}\left(r_{2}, q_{2}\right) \quad \text { and } \quad \pi_{n}\left(r_{1}, q\left(r_{1}\right)\right)=\pi_{n}\left(r_{2}, q_{2}\right) .
$$

Let $q_{i}:=q\left(r_{i}\right), \gamma_{i}:=\gamma\left(r_{i}, q_{i}\right), i \in\{1,2\}$. By the above one must have, step-by-step:

$$
\begin{aligned}
\pi_{n}\left(r_{1}, q_{1}\right)-\pi_{c}\left(r_{1}, q_{1}\right) & =\pi_{n}\left(r_{2}, q_{2}\right)-\pi_{c}\left(r_{2}, q_{2}\right) \\
\int_{0}^{\gamma_{1}} \int_{0}^{v} z f_{(12)}(v, z) d z d v & =\int_{0}^{\gamma_{2}} \int_{0}^{v} z f_{(12)}(v, z) d z d v \\
\gamma_{1} & =\gamma_{2}=: \gamma^{*} \\
0=\pi_{c}\left(r_{1}, q_{1}\right)-\pi_{c}\left(r_{2}, q_{2}\right) & =\left(r_{1}-r_{2}\right) \int_{\gamma^{*}}^{1} \int_{0}^{\gamma^{*}} f_{(12)}(v, z) d z d v \\
\gamma^{*} & =1 \\
\pi_{c}\left(r_{1}, q_{1}\right) & =0 .
\end{aligned}
$$

However, this cannot be an equilibrium, because by choosing $r=0$ type $c$ can assure himself an expected profit equal to $E\left(X_{(2: 2)}\right)$.

Proposition 5. The game has no partial pooling equilibrium with the following properties: type $n$ randomizes, and the single element that is common to the supports of the strategies of $c$ and $n$, which is denoted by $r^{*}$, is positive.

Proof. Suppose there is such an equilibrium in which the common element of the supports is denoted by $r^{*}>0$. Let $r_{n} \neq r^{*}$ be another element of the support of type $n$ 's mixed strategy. $r^{*}$

First notice that in equilibrium one has $q\left(r^{*}\right)<1$ because if $q\left(r^{*}\right)$ were equal to one, type $c$ could increase his payoff by setting $r=0$ in lieu of $r^{*}$.

In equilibrium type $n$ must be indifferent between choosing $r^{*}$ and $r_{n}$, which implies

$$
\left.\pi_{n}\left(r^{*}, q\left(r^{*}\right)\right)=\pi_{n}\left(r_{n}, 1\right)\right)=E\left(X_{(2: 2)}\right) .
$$

By (4) and the fact that $r \geq \hat{r} \Rightarrow \gamma(r, q)=1$, one has

$$
\pi_{n}\left(r^{*}, q\left(r^{*}\right)\right)= \begin{cases}E\left(X_{(2: 2)}\right) & \text { if } r^{*} \geq \hat{r} \\ E\left(X_{(2: 2)}\right)+\xi & \text { otherwise. }\end{cases}
$$

Let $q^{*}:=q\left(r^{*}\right), \gamma^{*}:=\gamma\left(r^{*}, q^{*}\right)$. In order to $\operatorname{sign} \xi$, recall that $r^{*}=\left(1-q^{*}\right) \gamma^{*}+q^{*} E\left(X \mid X \leq \gamma^{*}\right)$ by (1). Therefore, for all $r^{*} \in(0, \hat{r})$ :

$$
\begin{aligned}
\xi:= & r^{*} \int_{\gamma^{*}}^{1} \int_{0}^{\gamma^{*}} f_{(12)}(v, z) d z d v-\int_{\gamma^{*}}^{1} \int_{0}^{\gamma^{*}} z f_{(12)}(v, z) d z d v \\
= & 2 r^{*} F\left(\gamma^{*}\right)\left(1-F\left(\gamma^{*}\right)\right)-2 \int_{\gamma^{*}}^{1} \int_{0}^{\gamma^{*}} z f(z) f(v) d z d v \\
= & 2\left(\left(1-q^{*}\right) \gamma^{*}+q^{*} E\left(X \mid X \leq \gamma^{*}\right)\right) F\left(\gamma^{*}\right)\left(1-F\left(\gamma^{*}\right)\right) \\
& -2 E\left(X \mid X \leq \gamma^{*}\right) F\left(\gamma^{*}\right)\left(1-F\left(\gamma^{*}\right)\right) \\
= & 2\left(1-q^{*}\right)\left(\gamma^{*}-E\left(X \mid X \leq \gamma^{*}\right)\right) F\left(\gamma^{*}\right)\left(1-F\left(\gamma^{*}\right)\right)>0 .
\end{aligned}
$$

By (9)-(11) one must have $r^{*} \geq \hat{r}$. However, for all $r^{*} \geq \hat{r}$ one has $\gamma^{*}=1$, and hence $\pi_{c}\left(r^{*}, q^{*}\right)=0$. But then it is profitable for type $c$ to deviate to $r=0$ because he then earns the higher payoff $\pi_{c}(0, q)=E\left(X_{(2: 2)}\right)$, regardless of bidders' beliefs $q$. Therefore, the candidate mixed equilibrium is not an equilibrium. 
This result has a simple intuitive explanation. If there were such a partial pooling (mixed strategy) equilibrium, the payoff of type $n$ when he chooses an action $r_{n} \neq r^{*}$ (and thus is recognized as type $n$ with certainty) must be equal to $E\left(X_{(2: 2)}\right)$, by (7). Therefore, by the indifference condition of a mixed equilibrium, one must also have $\pi_{n}\left(r^{*}, q^{*}\right)=E\left(X_{(2: 2)}\right)$. But this is only possible if either $r^{*}=0$ (which is excluded by assumption) or $\gamma^{*}=1$, which implies $\pi_{c}\left(r^{*}, q^{*}\right)=0$. But then $r^{*}$ cannot be an equilibrium strategy because by choosing $r=0$ type $c$ can assure himself a higher payoff equal to $E\left(X_{(2: 2)}\right)$.

We conclude that the game may only have partial pooling equilibria in which either only type $c$ randomizes or $n$ randomizes and the one common element of the supports of equilibrium strategies is $r^{*}=0$. However, as we show next, if there is such an equilibrium, it violates the intuitive criterion.

\section{Equilibrium selection}

Proposition 6. Eliminating all (partial) pooling equilibria that violate the intuitive criterion yields the unique pooling equilibrium with $r^{*}=0$. In that equilibrium, commitment has no value.

Proof. We break down the assertion into two parts: 1) the pooling equilibrium with $r^{*}=0$ does not violate the intuitive criterion, and 2) all other pooling (or partial pooling) equilibria violate it.

1) Suppose there exists an $r^{\prime}>0$ from which type $c$ can benefit if he thus convinces bidders that he is type $c$, i.e., $\pi_{c}\left(r^{\prime}, 0\right)>\pi_{c}(0, q(0))=E\left(X_{(2: 2)}\right)$. Then, if type $n$ mimics $c$ and also plays $r^{\prime}$ and thus convinces bidders that he is type $c$, he benefits even more, because by (4), (3),

$$
\pi_{n}\left(r^{\prime}, 0\right) \geq \pi_{c}\left(r^{\prime}, 0\right)>E\left(X_{(2: 2)}\right)=\pi_{n}(0, q(0)) .
$$

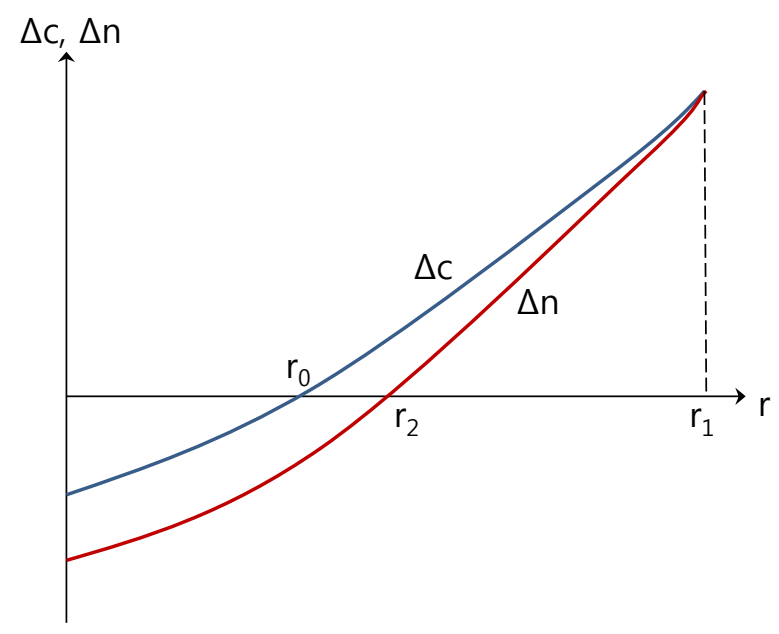

Figure 1: Elimination of implausible beliefs by the intuitive criterion

2) Consider any pooling (or partial pooling) equilibrium in which $r^{*}$ is the unique element of the intersection of the supports of strategies. We show below that there exists an action $r^{\prime}<r^{*}$ such that $\pi_{c}\left(r^{\prime}, 0\right)>\pi_{c}\left(r^{*}, q_{0}\right)$ and $\pi_{n}\left(r^{\prime}, 0\right)<\pi_{n}\left(r^{*}, q_{0}\right)$. Deviating to that action $r^{\prime}$ should convince bidders that the auctioneer is type $c$ because type $n$ cannot benefit from such a deviation even under the most favorable change of beliefs $q\left(r^{\prime}\right)=0$.

First notice that $q\left(r^{*}\right) \geq q_{0}>0$ because type $n$ does not randomize by Proposition 5 .

Let $\Delta_{i}(r):=\pi_{i}(r, 0)-\pi_{i}\left(r^{*}, q_{0}\right), i \in\{c, n\}$. These functions have the following properties, which are summarized in Figure 1 ; 
a) The functions intersect at $r_{1}:=\gamma\left(r^{*}, q_{0}\right)>r^{*}$, because $\Delta_{c}\left(r_{1}\right)=\Delta_{n}\left(r_{1}\right) \Rightarrow \gamma\left(r_{1}, 0\right)=\gamma\left(r^{*}, q_{0}\right)$ and $\gamma\left(r_{1}, 0\right)=r_{1}$ by (1).

b) $\Delta_{c}(0) \leq 0$, because $\pi_{c}(0,0)=E\left(X_{(2: 2)}\right) \leq \pi_{c}\left(r^{*}, q_{0}\right)$.

c) $\Delta_{c}\left(r^{*}\right)>0$, because $\pi_{c}(r, q)$ is strictly decreasing in $q$.

d) There exists an $r_{0} \in\left[0, r^{*}\right)$ at which $\Delta_{c}\left(r_{0}\right)=0$.

e) For all $r \in\left[0, r_{1}\right): \Delta_{c}(r)<\Delta_{n}(r)$, because $\gamma(r, 0)=r$ and hence

$$
\begin{aligned}
\partial_{r} \pi_{n}(r, 0) & =\partial_{r} \pi_{c}(r, 0)+\partial_{r}\left(\int_{0}^{r} \int_{0}^{v} z f_{(12)}(v, z) d z d v\right) \\
& >\partial_{r} \pi_{c}(r, 0) .
\end{aligned}
$$

From a)-e) it follows that there exists a nonempty interval (as illustrated by the interval $\left(r_{0}, r_{2}\right)$ in Figure 1 1 ) so that all $r^{\prime}$ chosen from that interval constitute a deviation that induces the asserted belief change.

Altogether, we thus arrive at the paradoxical conclusion:

Corollary 1. After eliminating implausible beliefs that violate the intuitive criterion, in equilibrium commitment has no value even if the prior probability of facing an uncommitted type of auctioneer is arbitrarily small.

\section{Discussion}

We mention that if the number of bidders is increased, the threshold level of bidder participation $\gamma$ tends to diminish. In other words, the probability of bidders' speculative behavior diminishes. Therefore, the speculation induced by imperfect commitment becomes less prominent when the number of bidders is large.

The above analysis of the open, ascending-bid English clock auction also applies to a second-price sealed bid auction, provided the reserve is defined as the minimum price a bidder has to pay if he wins. However, if the reserve is defined as a minimum bid, the analysis differs. In that case, all bidders will bid, even those with low valuations, but those whose valuation is higher than the reserve price but relatively close to it, will shade their bid, speculating on the event that the secondhighest bid is sufficiently below the reserve. Therefore, if the reserve is a minimum bid requirement the English clock auction and the second-price sealed-bid auction are not revenue equivalent.

\section{A Appendix: Supplement to the proof of Proposition 1}

Here we show that bidders' equilibrium strategy must be a cutoff strategy.

Consider a measurable set $X \subset[0,1]$. Define $m(X):=\int_{X} d F(x)$ and $\mu(X):=\int_{X} x d F(x) / m(X)$, i.e., $m(X)$ is the probability that a bidder's value belongs to $X$ and $\mu(X)$ the average of values that belong to $X$.

Lemma A.1. In any equilibrium, if bidder $i$ with value $v$ participates in the first round, then all types of $i$ with values greater than $v$ also participate in the first round.

Proof. Let $X_{1}$ be the set of the opponent's types that participate in the first round and $X_{0}$ be the complement of $X_{1}$. Also define

$$
\begin{aligned}
& X_{1}^{L}:=X_{1} \cap\{x \mid x \leq v\} \\
& X_{1}^{H}:=X_{1} \cap\{x \mid x>v\}
\end{aligned}
$$

\footnotetext{
${ }^{7}$ Figure 1 is actually an exact representation of the uniform distribution case.
} 


$$
\begin{aligned}
X_{0}^{L} & :=X_{0} \cap\{x \mid x<r\} \\
X_{0}^{H} & :=X_{0} \cap\{x \mid x \geq r\} \\
X_{0}^{H L} & :=X_{0}^{H} \cap\{x \mid x \leq v\} \\
X_{0}^{H H}: & =X_{0}^{H} \cap\{x \mid x>v\}
\end{aligned}
$$

Then the expected payoff of bidder $i$ with value $v$ if he participates in the first round $\left(U_{i}^{P}(v)\right)$ and that if he does not participate in the first round $\left(U_{i}^{N P}(v)\right)$ are

$$
\begin{aligned}
U_{i}^{P}(v) & =m\left(X_{0}\right)(v-r)+m\left(X_{1}^{L}\right)\left(v-\mu\left(X_{1}^{L}\right)\right) \\
U_{i}^{N P}(v) & =q\left[m\left(X_{0}^{L}\right)\left(v-\mu\left(X_{0}^{L}\right)\right)+m\left(X_{0}^{H L}\right)\left(v-\mu\left(X_{0}^{H L}\right)\right)\right]
\end{aligned}
$$

For some $v^{\prime}>v$ define $X_{1}^{H L}:=X_{1}^{H} \cap\left\{x \mid x \leq v^{\prime}\right\}$ and $X_{0}^{H H L}:=X_{0}^{H H} \cap\left\{x \mid x \leq v^{\prime}\right\}$. Then the expected payoff of bidder $i$ with value $v^{\prime}$ if he participates in the first round $\left(U_{i}^{P}\left(v^{\prime}\right)\right)$ and that if he does not participate in the first round $\left(U_{i}^{N P}\left(v^{\prime}\right)\right)$ are

$$
\begin{aligned}
U_{i}^{P}\left(v^{\prime}\right) & =m\left(X_{0}\right)\left(v^{\prime}-r\right)+m\left(X_{1}^{L}\right)\left(v^{\prime}-\mu\left(X_{1}^{L}\right)\right)+m\left(X_{1}^{H L}\right)\left(v^{\prime}-\mu\left(X_{1}^{H L}\right)\right) \\
U_{i}^{N P}\left(v^{\prime}\right) & =q\left[m\left(X_{0}^{L}\right)\left(v^{\prime}-\mu\left(X_{0}^{L}\right)\right)+m\left(X_{0}^{H L}\right)\left(v^{\prime}-\mu\left(X_{0}^{H L}\right)\right)+m\left(X_{0}^{H H L}\right)\left(v^{\prime}-\mu\left(X_{0}^{H H L}\right)\right)\right]
\end{aligned}
$$

Hence, we have

$$
\begin{aligned}
U_{i}^{P}\left(v^{\prime}\right) & =U_{i}^{P}(v)+\left(m\left(X_{0}\right)+m\left(X_{1}^{L}\right)\right)\left(v^{\prime}-v\right)+m\left(X_{1}^{H L}\right)\left(v^{\prime}-\mu\left(X_{1}^{H L}\right)\right) \\
U_{i}^{N P}\left(v^{\prime}\right) & =U_{i}^{N P}(v)+q\left[\left(m\left(X_{0}^{L}\right)+m\left(X_{0}^{H L}\right)\right)\left(v^{\prime}-v\right)+m\left(X_{0}^{H H L}\right)\left(v^{\prime}-\mu\left(X_{0}^{H H L}\right)\right)\right]
\end{aligned}
$$

Because $m\left(X_{0}\right) \geq m\left(X_{0}^{L}\right)+m\left(X_{0}^{H L}\right)+m\left(X_{0}^{H H L}\right), \mu\left(X_{0}^{H H L}\right)>v$, and $U_{i}^{P}(v) \geq U_{i}^{N P}(v)$, it follows that $U_{i}^{P}\left(v^{\prime}\right)>U_{i}^{N P}\left(v^{\prime}\right)$.

Corollary A.1. In any equilibrium, if bidder $i$ with value $v$ does not participate in the first round, then all types of $i$ with values less than $v$ do not participate in the first round either.

Corollary A.2. In equilibrium every bidder plays a cutoff strategy: All types of bidder $i$ with values $v<v_{i}^{*}$ do not participate in the first round, and all types of $i$ with values $v>v_{i}^{*}$ participate in the first round for some $v_{i}^{*} \in[r, 1]$.

\section{References}

Bagwell, K., 1995. Commitment and observability in games. Games and Economic Behavior 8, 271-280.

Caillaud, B., Mezzetti, C., 2004. Equilibrium reserve prices in sequential auctions. Journal of Economic Theory 117, 78-95.

Cho, I. K., Kreps, D., 1987. Signaling games and stable equilibria. Quarterly Journal of Economics $102,179-221$.

Haile, P., 2000. Partial pooling at the reserve price in auctions with resale opportunities. Games and Economic Behavior 33, 231-248.

Kreps, D., 1990. A Course in Microeconomic Theory. Princeton University Press.

McAfee, R. P., Vincent, D., 1997. Sequentially optimal auctions. Games and Economic Behavior $18,246-276$.

Van Damme, E., 1987. Stability and Perfection of Nash Equilibria. Springer-Verlag.

van Damme, E., Hurkens, S., 1997. Games with imperfectly observable commitment. Games and Economic Behavior 21, 282-308. 